\title{
Extensive characterization of magnetic microrods observed by optical microscopy
}

Authors:

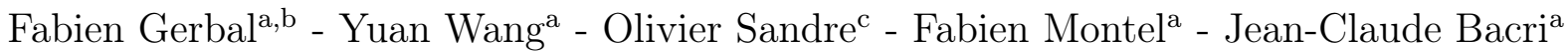

Authors affiliation:

a: Laboratoire Matière et Systèmes Complexes (MSC) UMR 7057 (CNRS) and Université Denis Diderot - Sorbonne Paris Cité, 10 rue Alice Domon et Léonie Duquet, 75013 Paris, France

b: Université Pierre et Marie Curie - Paris 6, Sorbonne Universités, 4 place Jussieu, 75252 Paris Cedex 05, France

c: CNRS and Université de Bordeaux, Laboratoire de Chimie des Polymères Organiques (LCPO), UMR 5629, F-33600 Pessac, France

Corresponding author:

Fabien Gerbal

fabien.gerbal@upmc.fr

Tel: +33 (0)1572762 88 - Fax: +33(0)157276211

Laboratoire MSC - UMR 7057

Université Denis Diderot,

Bâtiment Condorcet

10 rue Alice Domon et Léonie Duquet,

75205 Paris cedex 13, France

keywords:

characterization $\mid$ micro/nanorod $\mid$ micro/nanowire $\mid$ susceptibility | rigidity | stiffness $\mid$ superparamagnetic | 


\section{ABSTRACT}

The usage of micro or nanorods is steadily increasing in various applications from fundamental research to industry. Therefore their geometrical, mechanical and eventually magnetic properties need to be well determined. Here, using an optical microscope equipped with magnetic tweezers, we report an experimental procedure to obtain all these information on a single magnetic rod. In particular, we measured the magnetic susceptibility $\chi$ by analyzing the deformation of a rod submitted to a uniform magnetic field. To do so, we refined a theoretical model which takes into account the variation of $\chi$ with the internal field. We prove experimentally that this model yields consistent measures, at any value of field strength and incidence angle. From the combination of the different measurements, we also deduced the number of iron oxide nanoparticles which are embedded within the polymer matrix of the superparamagnetic rods under study.

A very broad research activity concerns micro and nanoscale magnetic rods which show fundamental interest in many applications such as surface coating reagents, actuators, bio-medical probes and so on [4]. Depending on their size, various strategies have been developed to synthesize them: near-micron radius or larger wires are made for instance from rapid quenching [22], whereas the probably most common strategy for preparing rods around tens of nanometers diameter consists in filling the pores of an etched template by electrochemical deposition of one or several metals [6]. Other groups realized nanorods by metallizing bacteria [14], by chaining particles together [17], or by embodying nanoparticles into an elastomer gel such as PDMS through lithographic technics [18]. Many applications for which these rods are designed require a well-characterized calibration of their properties, often at the level of the individual object rather than for a broad distribution.

We report the measurements of their length $L$, radius $r$, flexural rigidity $C$, and magnetic susceptibility $\chi$ performed on single individual rods by using a conventional optical microscope equipped with magnetic tweezers (ESI†, note 1 shows the complete scheme of all measurements). Whereas the measurement presented in the paper could apply to many types of magnetic rods, we studied $L \sim 10$-80 micron-long superparamagnetic rods with $r \sim 200-400 \mathrm{~nm}$ made of iron oxide nanoparticles (diameter $13 \mathrm{~nm}$ ) embedded into a polymer matrix $[3,7,11,12,20]$. We initially assessed each measurement independently: the super-resolutive optical model used to derive $r$ was compared to Atomic Force Microscopy (AFM) scans performed on the same rods; the measured value of $C$ was derived from the analysis of the thermal fluctuations [11]; $\chi$ was obtained from the measurement of 
the tip deflection of a magnetically-bent rod. To do so, we used a theoretical model (which takes into account the variation of $\chi(H)=\frac{M(H)}{H}$ with the field strength $H$ [12]) to describe the rod magnetoelastic deformation. We present an improved version of this model which applies well to rods of relatively low magnetic susceptibility such as the ones studied here. By comparison of the resulting $\chi$ value with the susceptibility $\chi^{v}$ of the particles which compose the rods (measured independently by vibrating sample magnetometry (VSM)), we deduced the volume fraction $\phi$ of the magnetic nanoparticles. We also report experiments which yield constant values for $\phi$, independently of the intensity and incidence angle of the field, thereby demonstrating the robustness of the model. Finally, the full analysis performed on 10 different rods showed consistent and relatively low dispersion of the values found for $\phi \sim 10 \%-30 \%$ considering the broad disparity of $\chi \sim 2.0-7.4$ (at $\mu_{0} H=4 \mathrm{mT}$ ) and $C \sim 10^{-18}-10^{-20} \mathrm{Jm}$.

The $\gamma-\mathrm{Fe}_{2} \mathrm{O}_{3}$ (maghemite) iron oxide nano-particles which compose the rods were synthesized by the aqueous coprecipitation route also called the Massart procedure, followed by a size sorting procedure [1]. Their nanometric size yields their superparamagnetic properties (each particle has a single Weiss magnetization domain) and their size disparity accounts for the divergence between the Langevin law and the magnetization curve of the particle ferrofluid suspension [5]. This result was confirmed by our own magnetization curves (ESI†, Fig. S2) of a ferrofluid suspension of negatively charged (citrated) nanoparticles (volume fraction $\phi^{v}=3.9 \%$ ) obtained by VSM using a home-made apparatus, from which was deduced the log-normal distribution of the particle diameters (10-16 nm range, mean=13 nm) [16]. Following an already published protocol [11], the rods were then prepared by dialysis of a solution of the nanoparticles mixed with positively charged polymers (poly(diallyldimethylammonium chloride)) while being exposed to a $\sim 250 \mathrm{mT}$ magnetic field. We used a microscopic setup equipped with magnetic tweezers designed to induce a uniform magnetic field on the sample (Fig. 1). We carefully calibrated the field, and checked that the influence of the gradient was negligible [11]. To prepare the samples, we flowed a 100x diluted rod solution into an observation chamber, so that some of the rods were found in a cantilevered configuration, i.e. with a small part of the rod stuck on an inner coverslip while the longest part remained far from any solid surface (Fig. 1B). After performing the measurements, we checked that the clamped boundary condition was fulfilled by controlling that the anchorage resisted to a large deformation induced by a magnet brought at vicinity of the sample. The rods were observed with a DMIRB inverted Leica microscope equipped with an apo plan 100x NA 1.3 objective and a Photometrics fx-Coolsnap cam- 
era (pixel size $\left.(4.65 \mu \mathrm{m})^{2}\right)$ either by bright field or by reflection microscopy (Fig. 1C). Home made softwares were written to automatically pilot the magnetic tweezers and the camera, track noisy motion of the sample and digitize the rod shape with a resolution of 2-20 $\mathrm{nm}$ [11].

Before performing the complete set of measurements on individual rods, we checked the validity of each of them. To characterize the rod geometry, the measure of $L \sim 30-80 \mu \mathrm{m}$ was straight forward on the images taken with the microscope, but the measure of $r \sim 300 \mathrm{~nm}$ was more challenging because of the optical diffraction limit. We thus developed a theoretical model to compute the rod reflection intensity profile: $I_{r}(x)=I_{0}+I_{1} \int_{-r}^{r} \exp \left[-\frac{\left.\left(s-x-x_{0}\right)\right)^{2}}{2 \sigma_{x y}{ }^{2}}\right] 2 r \sqrt{1-\left(\frac{s}{r}\right)^{2}} d s$, where $I_{0}$ is the background intensity, $I_{1}$ an effective intensity, $\sigma_{x y}$ is a function of the wavelength and the objective numerical aperture, and $x$ the abscissa along the rod cross section (ESI $\dagger$, note 1 ). The value of $r$ was then obtained from the fit of this function to the experimental intensity measured at each rod cross-sections (Fig. 1C). We checked our model by comparing its result to the value of $r$ measured by AFM on the same sample rods (ESI $\dagger$, note 1 and Fig. S3). We found that the uncertainty of this optical measurement was of $\sim 30 \mathrm{~nm}$, which is also the typical standard deviation of $r$ along the rod.

The mechanical characterization of the rod was previously fully described [11] and consisted of acquiring 900 images of the rod to analyze its thermal fluctuations, from which were deduced the rod persistence length $L p$, its bending modulus $C=L_{p} / k_{B} T$ ( $k_{B} T$ is the thermal energy) and its Young modulus $Y=\frac{C}{4 \pi r^{4}}$.

The magnetic susceptibility $\chi$ of the rod material (defined as $\chi=\frac{M}{H}$, where $M$ is the magnetization and $H$ the field inside the material) was deduced from magnetoelastic experiments in which we analyzed the deflection of the rod deformed by a uniform field. During such magnetic deformation, the magnetic torque per unit volume $\Gamma_{m}=\left\|\vec{M} \wedge \overrightarrow{B_{0}}\right\|\left(\overrightarrow{B_{0}}\right.$ is the uniform induction field $)$ acting on the rod is counter-balanced by the elastic restoring torque $\frac{C}{R}, R$ being the local radius of curvature. As shown in ESI $\dagger$, note $2, \Gamma_{m}=\Delta \chi \sin (2 \alpha) \frac{\pi r^{2} B_{0}^{2}}{2 \mu_{0}}$, where $\mu_{0}$ is the vacuum permeability, $\alpha$ the angle of $\overrightarrow{B_{0}}$ with respect to the rod main axis and $\Delta \chi$ a function of $\chi$ and of the local components of the field $\vec{H}$ in the rod. Thus, the measurement of the rod deflection $\delta$ (Fig. 2A) may yield either one of the parameter $C$ or $\chi$ when the other one is known. However, a difficulty arises from the fact that $\chi$ depends on $H$. This can be directly observed on the magnetization curve of the nanoparticles before they were embedded in the rods (ESI† Fig. S2) which shows deviation from linearity even at very low field ( $\chi^{v}$ varies by more than a factor of 2 between 2 and $10 \mathrm{mT}$ ). Consistently, previously detailed [11] viscous drag versus magnetic torque (VD/MT) experiments (in which the magnetically 
driven rotational kinetics of free rods in solution is analyzed), also showed that the rod material susceptibility $\chi$ depends on $H$ and is proportional to $\chi^{v}$, the constant ratio $\chi / \chi^{v}$ being simply the ratio of the magnetic nanoparticle volume fraction within the rod and in the initial ferrofluid $\phi / \phi^{v}$.

Thus, to analyze the magnetoelastic experiments, we modified a theoretical model [12] to study the magnetoelastic deformation of low susceptibility rods. Although this model is only detailed here (ESI†, note 2), we showed that it matched accurately the shape of the deformed rods [11]. This model takes into account the dependence of $\chi$ with $H$, a variability which is often neglected $[2,7,9,20]$. Here, we also report experiments aimed at controlling that this model yields a constant measure of $\phi$ independently of the field incidence and strength (Fig. 2). Fig. 2B shows experiments on a 33.1 $\mu \mathrm{m}$ long rod oriented at various incidence angles $\theta_{0}$ from 0 to $60^{\circ}$ in a constant applied field. The deflection $\delta\left(\theta_{0}\right)$ (Fig. 2A) varies accordingly to our theoretical model: when $\theta_{0}$ varies, so does the internal field $H$ (ESI†, Fig S4). and we found that the variation of the measured $\chi(H)$ matches the data $\chi^{v}(H)$. Consequently the comparison yielded a fairly constant value for $\phi$ (within $\pm 2 \%$ ). This experiment and the theoretical curves shown on Fig. 2A also suggested that the best values at which these deflection measurements should be performed were $\theta_{0} \sim 30-50^{\circ}$ : at these angles, the deflection $\delta$ reaches a maximum plateau when $B_{0}$ is below the magnetoelastic buckling field [12].

Next, at a constant incidence $\theta_{0}=35 \pm 0.1^{\circ}$, we studied the effect of the field intensity on the rod deflection (Fig. 2C). We monitored 130 deformations of a $46.4 \mu \mathrm{m}$ long rod submitted to an incrementally increased intensity of the external induction field from 0 to $7 \mathrm{mT}$. The fields was successively turned on during $500 \mathrm{~ms}$ (a sufficient time to reach the equilibrium configuration) and off for the same amount of time to monitor the rod returning to its undeformed state. Excepted the thermal fluctuations, the deformations were reproducible. They did not increase incrementally, an effect which could have occurred in the case of remanent magnetization, thus confirming the superparamagnetism of the rod. During this experiment, the rod deflected by a maximum of $1.6 \mu \mathrm{m}$ (corresponding to a deviation of $2^{\circ}$ ), and the induced longitudinal field $\mu_{0} H_{0 \|}$ ranged from 0 to 4 $\mathrm{mT}$. The analysis of the deflection according to the theoretical model showed that $\chi\left(H_{0 \|}\right)$ varied by more than a factor of two, but remained proportional to $\chi^{v}\left(H_{0 \|}\right)$. The values found for $\chi$ below 2 mT (for which no VSM data are available) also appeared consistent with the expected shape of the magnetic susceptibility curve. The deduced measure of $\phi$ was found to vary by less than $10 \%$ and

\footnotetext{
${ }^{*}$ for non-interacting and uniformly magnetized nanoparticles such as used here, the magnetization (and therefore the susceptibility) is simply proportional to the volume of magnetic matter.
} 
yielded a mean value of $\langle\phi\rangle=15 \% \pm 0.8 .^{\dagger}$ In contrast, if $\chi$ is assumed constant, $\phi$ varies by a factor of two when deduced from these measurements.

We then proceeded to the full characterization of ten individual rods. All came from the same synthesis batch. Once $L, r$ and $C$ were determined as described above, the measurement of $\chi$ consisted in submitting the rod to $\sim 1$ s long pulses of magnetic field applied at a $35^{\circ}$ incidence. The field intensity was varied from $\sim 2$ to $\sim 8 \mathrm{mT}$ to check the constancy of $\phi$ for a given rod. At each field intensity, the measurements were repeated 5 times to averaged out the errors mostly due to the thermal fluctuations ( 1/10 of the magnetic deflections) (ESI $\dagger$, Fig. S5A and Movie 1). The deflections always remained small $\left(\delta \sim 1 \mu \mathrm{m}, \theta_{L} \lesssim 1^{\circ}\right)$, which also ensured that the system was probed in the linear elastic regime: for larger deformations, we observed a plastic behavior (ESI $\dagger$, Fig. S5b). To monitor small deformations, we took advantage of our super-resolutive image analysis algorithm [11] to measure the rod centerline from the microscopy images with a precision of a few tens of nanometers (Movie 1).

Table 1 shows the results for 10 rods selected over nearly 100 studied samples. Reasons for rejections were insufficiently straight or badly anchored rods, or uncontrolled vibrations of the sample (noise) which prevented the detection of the thermal fluctuations. The selected rods were homogeneous in length because we chose the longest to allow a fine detection of the thermal fluctuations. This selection might also explain the small dispersion in thickness as $r$ varies only from 0.3 to 0.4 $\mu \mathrm{m}$. By contrast the flexural rigidity was extremely variable, ranging from 21.5 to $536 \times 10^{-21} \mathrm{Jm}$, corresponding to a persistence length from 5 to $130 \mathrm{~m}$ and a Young modulus of 2 to $60 \mathrm{MPa}$. The measured susceptibility (for $B_{0}=4 \mathrm{mT}$ ) was found to vary by a factor of $3(<\chi>=3.8 \pm 1.7)$ as the volume fraction $(\langle\phi\rangle=14.7 \pm 6.7)$ and the mean particle number per rod cross-section: $144 \pm 59 .{ }^{\ddagger}$

In these measurements, the final precision of only $25-50 \%$ on $\chi$ and $\phi$ mostly arises from the uncertainty on $C$, due to the difficulty to detect the small fluctuations of the rods $(\sim 10-100$ $\mathrm{nm}[11])$. However, this precision was sufficient to display the large disparity of the rod characteristics, unreported by the previous study on the stiffness of such rods [2] which contained a single measure. This surprisingly high dispersion is corroborated by several observations: (i) several previous papers concerning this type of rods also reported a large distribution of their properties such as their lognormal distribution of lengths [9] or as their susceptibility [7] which was plotted on a logarithmic

\footnotetext{
${ }^{\dagger}$ The standard deviation of $0.8 \%$ only accounts for the variability of the measures and not for the uncertainty of the other previously independently determined parameters (see Table 1).

$\ddagger$ The uncertainty for these numbers is the standard deviation of the measures on the 10 rods.
} 
scale; (ii) the VD/MT experiments performed on ten other rods gave a similar range of values for $\chi=4.6 \pm 1.2$ (for $B_{0}=4 \mathrm{mT}$ ), which consistently yielded a similar result for $\langle\phi\rangle=17.8 \pm 4.5 \%$ [11]; (iii) the alternative analysis of the magnetoelastic experiments (in which $\chi$ is set from the VD/MT experiments and $C$ is looked for) confirmed the values of $C$ determined by the thermal fluctuation method [11], and in particular (iv), the fact that despite their large variation and the complete independence of their measurements, the combination of values found for $C$ and $\chi$ yielded consistent results for $\phi=14.7 \pm 6.7 \%$ which falls into a narrow acceptable range of values: from electron micrographs [20], it has to be above $10 \%$ but also below $50 \%$, the fraction of randomly close-packed spheres. By comparison, a small angle neutron scattering study on isotropic aggregates made of different kind of polymers and smaller nanoparticles yielded a mean value for $\phi=38 \%$ [8]. In the present study, the large dispersion of values highlights the advantage of fully characterizing each rod individually: if we had only known a mean value for $C$ from a distribution of rods, the magnetoelastic experiments would have yielded results for $\chi$ ranging from 0.6 to 13 and inconsistent values of $\phi$ from 2.3 to $60 \%$ !

This study shows that the number of nanoparticles per rod cross-section is relatively small (Fig. 3), thus indicating that rods with a higher concentration of particles could be synthesized to obtain a greater magnetic susceptibility. This information on the microscopic structure also suggests that the rod stiffness is mostly dictated by the polymer matrix. A putative explanation of the rod stiffness variability is that fluctuations in numbers of particles from one rod to another yield even larger fluctuations of the concentration in polymer chains $c$, which adsorb onto the particles. This must induce variable elastic properties according to the scaling law of a polymer mesh $Y \propto c^{2.2}[15]$. For future uses, a sorting or a more selective method to produce the rods more homogeneously needs to be developed. Meanwhile, individual rod characterization as described in the paper may be required for many applications. We also believe that insights could be gained on numerous other types of magnetic microrods from the techniques we have introduced here.

\section{ACKNOWLEDGMENTS}

The authors thank M. Devaud for fruitful discussions and his patient proof-reading of the manuscript. 


\section{REFERENCES}

[1] J. C. Bacri, R. Perzynski, D. Salin, V. Cabuil, and R. Massart. Magnetic colloidal properties of ionic ferrofluids. Journal of Magnetism and Magnetic Materials, 62(1):36-46, 1986.

[2] L. Chevry and J.-F. Berret. Sub-piconewton force detection using micron-size wire deflections. RSC Advances, 3(38):17254, 2013.

[3] Rémy Colin, Loudjy Chevry, Jean-François Berret, and Bérengère Abou. Rotational microrheology of Maxwell fluids using micron-sized wires. Soft matter, 10(8):1167-73, 2014.

[4] Rupesh S. Devan, Ranjit A. Patil, Jin Han Lin, and Yuan Ron Ma. One-dimensional metal-oxide nanostructures: Recent developments in synthesis, characterization, and applications. Advanced Functional Materials, 22(16):3326-3370, 2012.

[5] W. C. Elmore. The Magnetization of Ferromagnetic Colloids. Physical Review, 54(12):10921095, dec 1938.

[6] A Fert and L Piraux. Magnetic nanowires. J. Magn. Magn. Mat., 200:338-358, 1999.

[7] J. Fresnais, J. F. Berret, B. Frka-Petesic, O. Sandre, and R. Perzynski. Electrostatic co-assembly of iron oxide nanoparticles and polymers: Towards the generation of highly persistent superparamagnetic nanorods. Advanced Materials, 20(20):3877-3881, 2008.

[8] J. Fresnais, J. F. Berret, L. Qi, J. P. Chapel, J. C. Castaing, O. Sandre, B. Frka-Petesic, R. Perzynski, J. Oberdisse, and F. Cousin. Universal scattering behavior of coassembled nanoparticle-polymer clusters. Physical Review E - Statistical, Nonlinear, and Soft Matter Physics, 78(4):20-23, 2008.

[9] B. Frka-Petesic, K. Erglis, J. F. Berret, A. Cebers, V. Dupuis, J. Fresnais, O. Sandre, and R. Perzynski. Dynamics of paramagnetic nanostructured rods under rotating field. Journal of Magnetism and Magnetic Materials, 323(10):1309-1313, 2011.

[10] F. Gazeau, E. Dubois, J. C. Bacri, F. Boue, A. Cebers, and R. Perzynski. Anisotropy of the structure factor of magnetic fluids under a field probed by small-angle neutron scattering. Physical Review E - Statistical, Nonlinear, and Soft Matter Physics, 65(3):1-15, 2002. 
[11] Fabien Gerbal and Yuan Wang. Optical detection of nanometric thermal fluctuations to measure the stiffness of rigid superparamagnetic microrods. Proceedings of the National Academy of Sciences, 114(10):2456-2461, mar 2017.

[12] Fabien Gerbal, Yuan Wang, Florian Lyonnet, Jean-claude Bacri, Thierry Hocquet, and Martin Devaud. A refined theory of magnetoelastic buckling matches experiments with ferromagnetic and superparamagnetic rods. Proceedings of the National Academy of Sciences, 112(23):71357140, jun 2015.

[13] L.D. Landau and E.M. Lifshitz. Theory of Elasticity. Pergamon press, Oxford, 2nd editio edition, 1970.

[14] Xin Liang, Jianhua Liu, Songmei Li, Yu Mei, and Wang Yanqing. Magnetic and mechanical properties of micro/nano particles prepared by metallizing rod-shaped bacteria. Materials Letters, 62(17-18):2999-3002, 2008.

[15] F.C. MacKintosh, J Käs, and P.A. Janmey. Elasticity of Semiflexible Biopolymer Networks. Physical Review Letters, 75(24):4425-4429, 1995.

[16] O. Sandre, J. Browaeys, R. Perzynski, J.-C. Bacri, V. Cabuil, and R. Rosensweig. Assembly of microscopic highly magnetic droplets: Magnetic alignment versus viscous drag. Physical Review E, 59(2):1736-1746, 1999.

[17] Harpreet Singh, Paul E. Laibinis, and T. Alan Hatton. Synthesis of flexible magnetic nanowires of permanently linked core-shell magnetic beads tethered to a glass surface patterned by microcontact printing. Nano Letters, 5(11):2149-2154, 2005.

[18] Joe W Tavacoli, Pierre Bauër, Marc Fermigier, Denis Bartolo, Julien Heuvingh, and Olivia du Roure. The fabrication and directed self-assembly of micron-sized superparamagnetic nonspherical particles. Soft Matter, 9(38):9103-9110, 2013.

[19] D. Thomann, D. R. Rines, P. K. Sorger, and G. Danuser. Automatic fluorescent tag detection in 3D with super-resolution: Application to the analysis of chromosome movement. Journal of Microscopy, 208(1):49-64, 2002. 
[20] M. Yan, J. Fresnais, S. Sekar, J. P. Chapel, and J. F. Berret. Magnetic nanowires generated via the waterborne desalting transition pathway. ACS Applied Materials and Interfaces, 3(4):1049$1054,2011$.

[21] B Zhang and J Enninga. Automated super-resolution detection of fluorescent rods in 2D. Biomedical Imaging: Nano to Macro, 2006. 3rd IEEE International Symposium on, pages 1-4, 2006.

[22] Valentina Zhukova, Mihail Ipatov, and Arcady Zhukov. Thin magnetically soft wires for magnetic microsensors. Sensors, 9(11):9216-9240, 2009. 


\section{FIGURES}
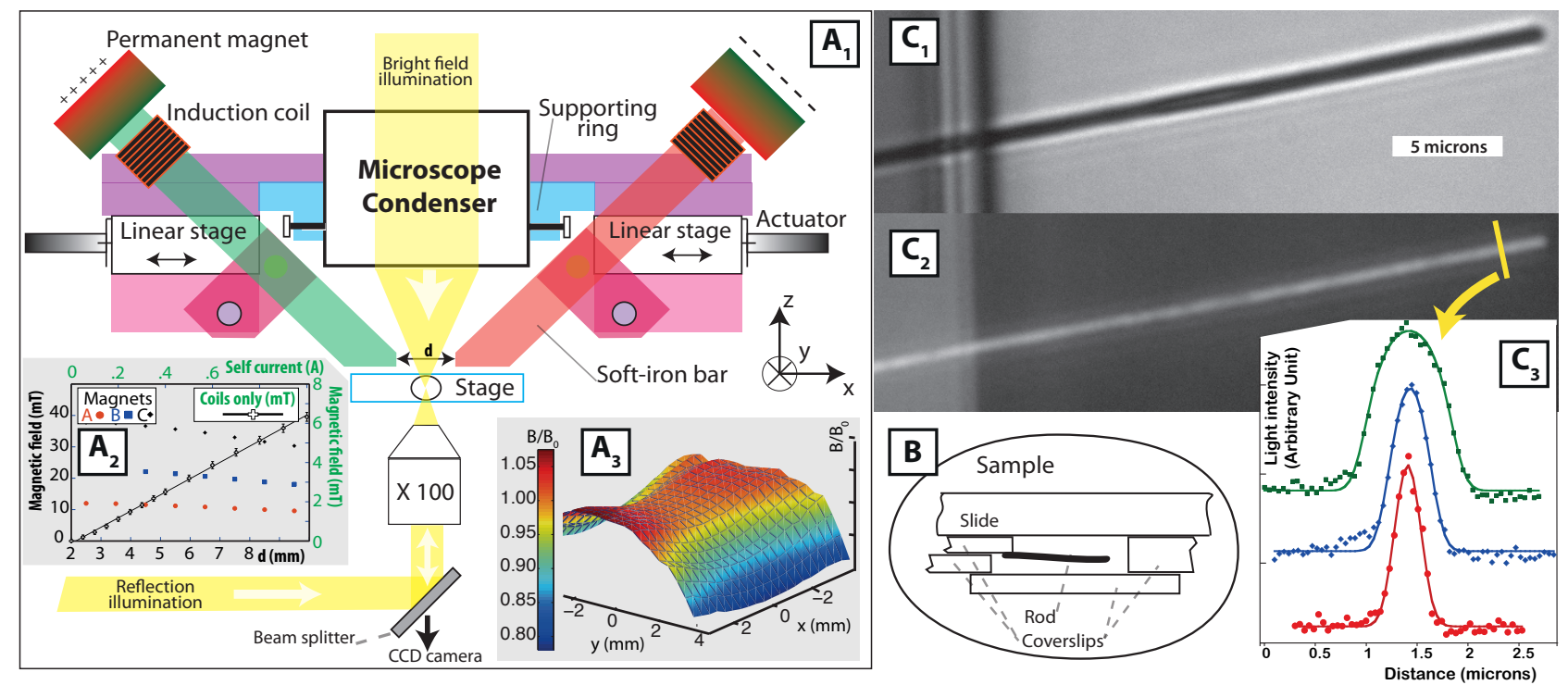

Fig. 1 Experimental setup and rod images. $\left(A_{1}\right)$ Microscope setup equipped with magnetic tweezers. The sample may be observed by standard bright field of by reflection microscopy by use of a beam splitter. Set on a ring attached to the condenser of the inverted microscope, the magnetic device is free to rotate in the $(\mathrm{x}, \mathrm{y})$ plane perpendicular to the microscope light path. It consists of two 7 $\mathrm{cm}$ diameter soft iron bars, planed at one end so they can be brought in the vicinity of the sample. They are mounted on actuated stages in order to vary the distance $d$ between their tips (precision $\sim 10 \mu \mathrm{m})$. The magnetic field is induced by permanent magnets or by induction coils at the remote end of these bars. The axial symmetry of the system insures that the field in the midperpendicular plane is horizontal. The magnets were used to demagnetize the bar before each experiments, and fine-tuned magnetic field could be generated by use of the coils (powered by a computer-controlled DC generator) without producing mechanical disturbances. $\left(\mathrm{A}_{2}\right)$ Calibration of the $\mathrm{x}$-component of the field at the expected position of the rod, for (i) a set of three magnets as a function of $d$ (axis in black) and (ii) as a function of the current in the coils (axis in green), for $d=2.5 \mathrm{~mm}$ and without magnet. The $\sim 2 \%$ error bars are mostly due to the uncertainty on the sample position along $z(\sim 10$ $\mu \mathrm{m})$ and the weak vertical field gradient $\left(\partial B_{x} / \partial z \sim 0.6 \mathrm{~T} / \mathrm{m}\right) .\left(\mathrm{A}_{3}\right) 3 \mathrm{D}$ representation of the spatial variation of the $\mathrm{x}$-component of the field between the tips of the iron bars, measured at the expected height of the rod. (B) Cross section of the sample cell made of various thickness coverslips glued together. The rod water solution was flowed into the cells and left for about 15-30' to sediment on the hang-over made by the inner coverslip. The sample was then set upside-down on the microscope stage. $\left(\mathrm{C}_{1}\right)$ Bright field image of a rod stuck at one extremity (left side) on the inner coverslip. Scale 
bar $=5 \mu \mathrm{m} .\left(\mathrm{C}_{2}\right)$ : Reflection image of the same rod. $\left(\mathrm{C}_{3}\right)$ shows three typical grey intensity profiles along a rod cross-section (such as indicated by the yellow line on $\mathrm{C}_{2}$ ) fitted by our theoretical model to measure the radius (yielding $r=148 \pm 8,386 \pm 7$ and $476 \pm 6 \mathrm{~nm}$ respectively for the red, blue and green curves). The red curve is taken from the rod shown on $\mathrm{C}_{2}$. 

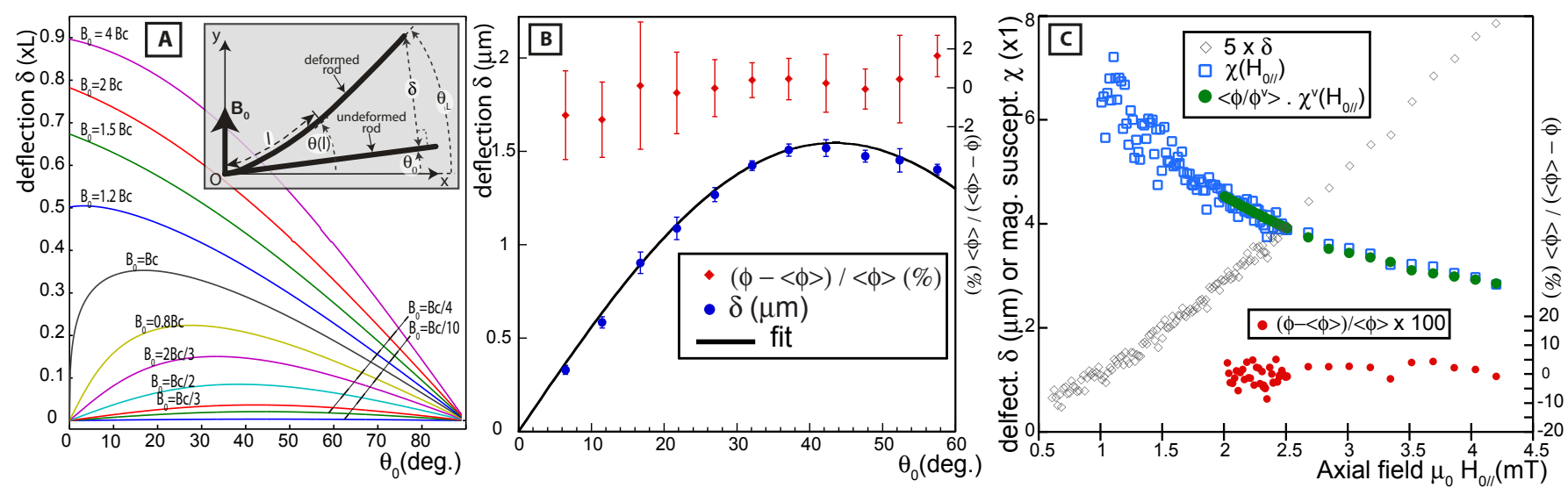

Fig. 2 Magnetoelastic experiments. (A) Inset: notations used to describe the magnetoelastic experiments, indicating in particular the deflection $\delta$ of the rod tip. Main curve: theoretical computations of $\delta$ using the "axial model" for a cantilevered rod of length $L=1$ as a function of the incidence angle $\theta_{0}$ of the field. Each curve corresponds to a magnetic induction intensity $B_{0}$ given relatively to the magnetoelastic buckling critical field $B_{c}$. For all curves such that $B_{0} \leqslant B_{c}$ the deflection is close to a maximum when $\theta_{0} \sim 35^{\circ}-50^{\circ}$. (B) Experimental data with a $33.1 \pm 0.05 \mu \mathrm{m}$ long rod $\left(C=1.6 \times 10^{-20} \mathrm{Jm}\right)$ probed at 11 incidence angles with $B_{0}=4.8 \pm 0.15 \mathrm{mT}$. The deflection $\delta$ (blue dots) is in excellent agreement with the theoretical curve of the axial model (solid black line) which corresponds to the " $B_{0}=B_{c} / 4$ " curve of (A). The error bars indicate the dispersion of the 5 measures taken at each angle. Red diamonds (scale on the right y-axis) are $\frac{\phi-\langle\phi\rangle}{\langle\phi\rangle}$ : the variation of the volume fraction $\phi$ (relatively to the mean of all values $\langle\phi\rangle$ ), computed from $\delta$ (see text and ESI $\dagger$, note 2 ). The variation remain below $\pm 2 \%$, indicating the independence of the measurement with $\theta_{0}$. (C) Analysis of the deflection of a cantilevered microrod $(r=0.34 \pm 0.04 \mu \mathrm{m}, L=46.37 \pm 0.05 \mu \mathrm{m}$, $\left.C=300 \pm 80 \times 10^{-21} \mathrm{Jm}\right)$ bent by an incrementally increased intensity of the magnetic induction field applied at $\theta_{0}=35^{\circ}$. The field $B_{0}$ varies from 0 to $4 \mathrm{mT}$ by $0.027 \mathrm{mT}$ steps and by $0.27 \mathrm{mT}$ steps from 4 to $7 \mathrm{mT}$ to reduce heating of the induction coils. The following quantities are plotted as a function of $B_{0 \|}=\mu_{0} H_{0 \|}$ (the projection of the field along the direction of the rod tip): 1(along the left-axis) $5 \times \delta$ (black diamonds) from which are computed $\chi\left(H_{0 \|}\right)$ (blue squares), and $<\phi / \phi^{v}>\times \chi^{v}\left(H_{0 \|}\right)$ (green dots) the magnetic susceptibility of the ferrofluid nanoparticles extrapolated at the mean volume fraction $\langle\phi\rangle$ of the particles in the the rod (VSM data are available only above $2 \mathrm{mT}$ ), and 2-(along the right-axis) $\frac{\phi-\langle\phi\rangle}{\langle\phi\rangle}$ (filled red circles) as in (B). The transverse field $\mu_{0} H_{\perp}$ (not plotted) varies from 0.11 to $1.5 \mathrm{mT}$ and is taken into account for the computations of $\phi$. 


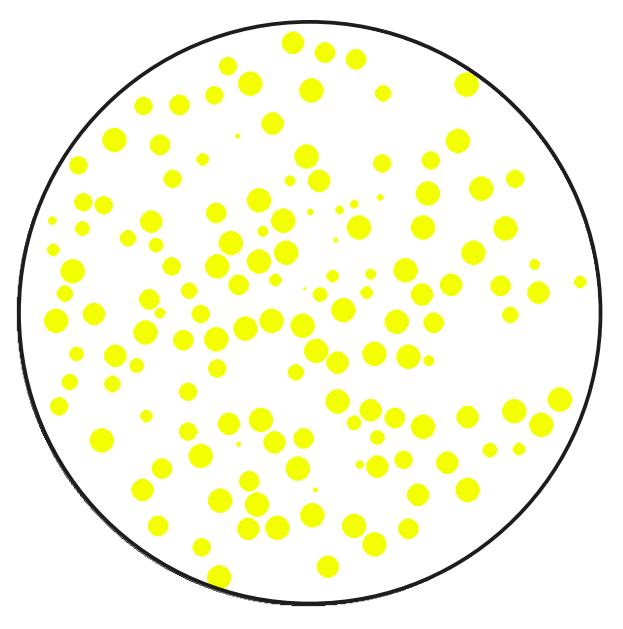

Fig. 3 Simulation of 144 nanoparticles $(\varnothing=13 \mathrm{~nm}$ ) (mean value found for the 10 analyzed rods detailed Table 1) randomly distributed in a cross-section of a rod of radius $r=300 \mathrm{~nm}$. 
Table 1 - Results for 10 rods

\begin{tabular}{|c|c|c|c|c|c|c|c|c|}
\hline N. & $\overline{\mathrm{L}(\mu m)}$ & $\overline{\mathrm{r}(\mu m)}$ & $\overline{\mathrm{C}\left(\mathrm{x} 10^{-21} \mathrm{Jm}\right)}$ & $\mathrm{Y}(\mathrm{MPa})$ & 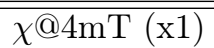 & $\overline{\phi \phi(\%)}$ & Part./rod (x1000) & Part./Cr.-Sec.(x1) \\
\hline 1 & $34.36 \pm 0.05$ & $0.316 \pm 0.027$ & $21.5 \pm 4$ & $2.7 \pm 1.5$ & $2.4 \pm 0.9$ & $9.2 \pm 3.4$ & $108 \pm 59$ & $82 \pm 45$ \\
\hline 2 & $26.83 \pm 0.05$ & $0.302 \pm 0.001$ & $38.1 \pm 16$ & $5.8 \pm 3.1$ & $4.1 \pm 1.7$ & $15.8 \pm 6.6$ & $132 \pm 66$ & $128 \pm 64$ \\
\hline 3 & $44.92 \pm 0.05$ & $0.366 \pm 0.024$ & $398 \pm 74$ & $28.3 \pm 12.6$ & $3.0 \pm 1.0$ & $11.4 \pm 4.0$ & $234 \pm 115$ & $135 \pm 67$ \\
\hline 4 & $35.94 \pm 0.05$ & $0.372 \pm 0.027$ & $76.7 \pm 14.3$ & $5.1 \pm 2.4$ & $2.00 \pm 0.7$ & $7.7 \pm 2.7$ & $130 \pm 67$ & $94 \pm 49$ \\
\hline 5 & $54.17 \pm 0.05$ & $0.410 \pm 0.080$ & $42.8 \pm 8.0$ & $1.9 \pm 1.9$ & $2.5 \pm 1.4$ & $9.75 \pm 5.2$ & $303 \pm 281$ & $145 \pm 135$ \\
\hline 6 & $42.41 \pm 0.05$ & $0.320 \pm 0.013$ & $448 \pm 84$ & $54 \pm 19$ & $7.4 \pm 2.4$ & $28.5 \pm 9.1$ & $422 \pm 174$ & $259 \pm 107$ \\
\hline 7 & $55.70 \pm 0.05$ & $0.330 \pm 0.040$ & $536 \pm 100$ & $58 \pm 39$ & $5.3 \pm 2.4$ & $20.3 \pm 9.4$ & $420 \pm 299$ & $196 \pm 140$ \\
\hline 8 & $25.52 \pm 0.05$ & $0.314 \pm 0.024$ & $154.0 \pm 29$ & $20 \pm 10$ & $3.4 \pm 1.3$ & $13.4 \pm 5.0$ & $115 \pm 62$ & $117 \pm 64$ \\
\hline 9 & $42.31 \pm 0.05$ & $0.301 \pm 0.014$ & $30.3 \pm 5.7$ & $4.7 \pm 1.8$ & $2.6 \pm 0.9$ & $10.0 \pm 3.6$ & $131 \pm 60$ & $81 \pm 37$ \\
\hline 10 & $36.41 \pm 0.05$ & $0.331 \pm 0.037$ & $158 \pm 30$ & $16.8 \pm 11$ & $5.5 \pm 2.3$ & $21.0 \pm 8.7$ & $285 \pm 185$ & $204 \pm 132$ \\
\hline mean & 39.86 & 0.336 & 190.4 & 19.8 & 3.8 & 14.7 & 228 & 144 \\
\hline $\mathrm{SD}$ & 10.15 & 0.035 & 196 & 21 & 1.7 & 6.7 & 124 & 59 \\
\hline
\end{tabular}

Measured parameters of 10 microrods from the same batch. The standard deviation for $L$ indicates the uncertainty of the measurement (estimated as a pixel size at each rod end). For $r$, the value is the mean $\pm \mathrm{SD}$ of the variation of the measure along the rod (the precision of a single measure being $30 \mathrm{~nm})$. For $C, \chi$ and $\phi$ the value is provided with the experimental uncertainty of the measurements. From these values, we estimate the total number of particles in each rod and per cross-section. The last two lines are the mean and SD of each parameter for the 10 analyzed rods. 


\section{ELECTRONIC SUPPLEMENTARY INFORMATION}

\section{Extensive characterization of magnetic microrods observed by optical microscopy}

F. Gerbal, Y. Wang, O. Sandre, F. Montel, JC. Bacri

ESI, Figure. S1: General scheme of the experiments

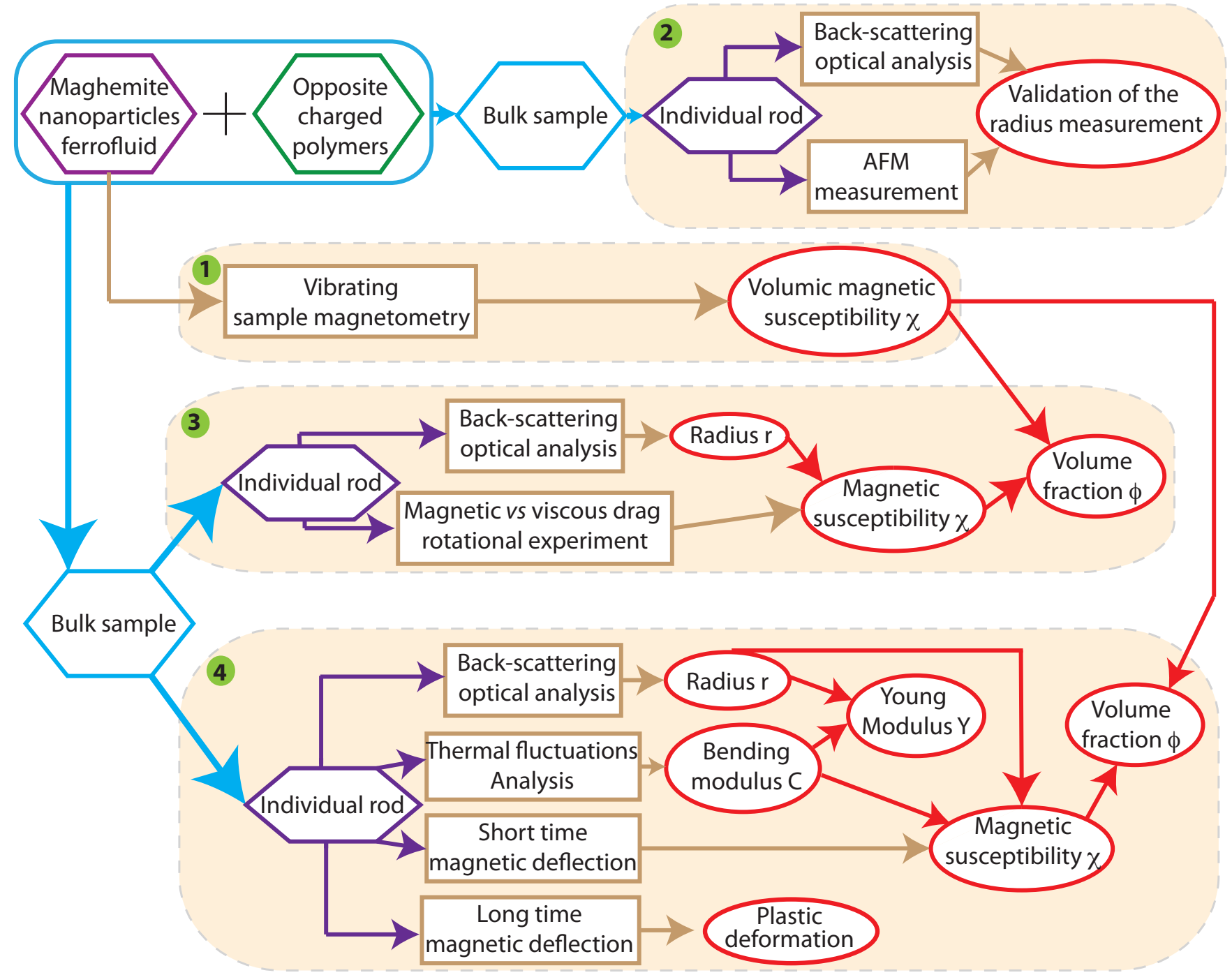

Fig. S1 General scheme of the experiments. The four groups of experiments explicit what are the measurements realized on the same rod, or on the same bulk sample (hexagons). Some variables (red round circles) are directly deduced by some measurements (squared boxes) or from a combination of them. 
ESI, Figure S2: Magnetization curves of the rod nanoparticles

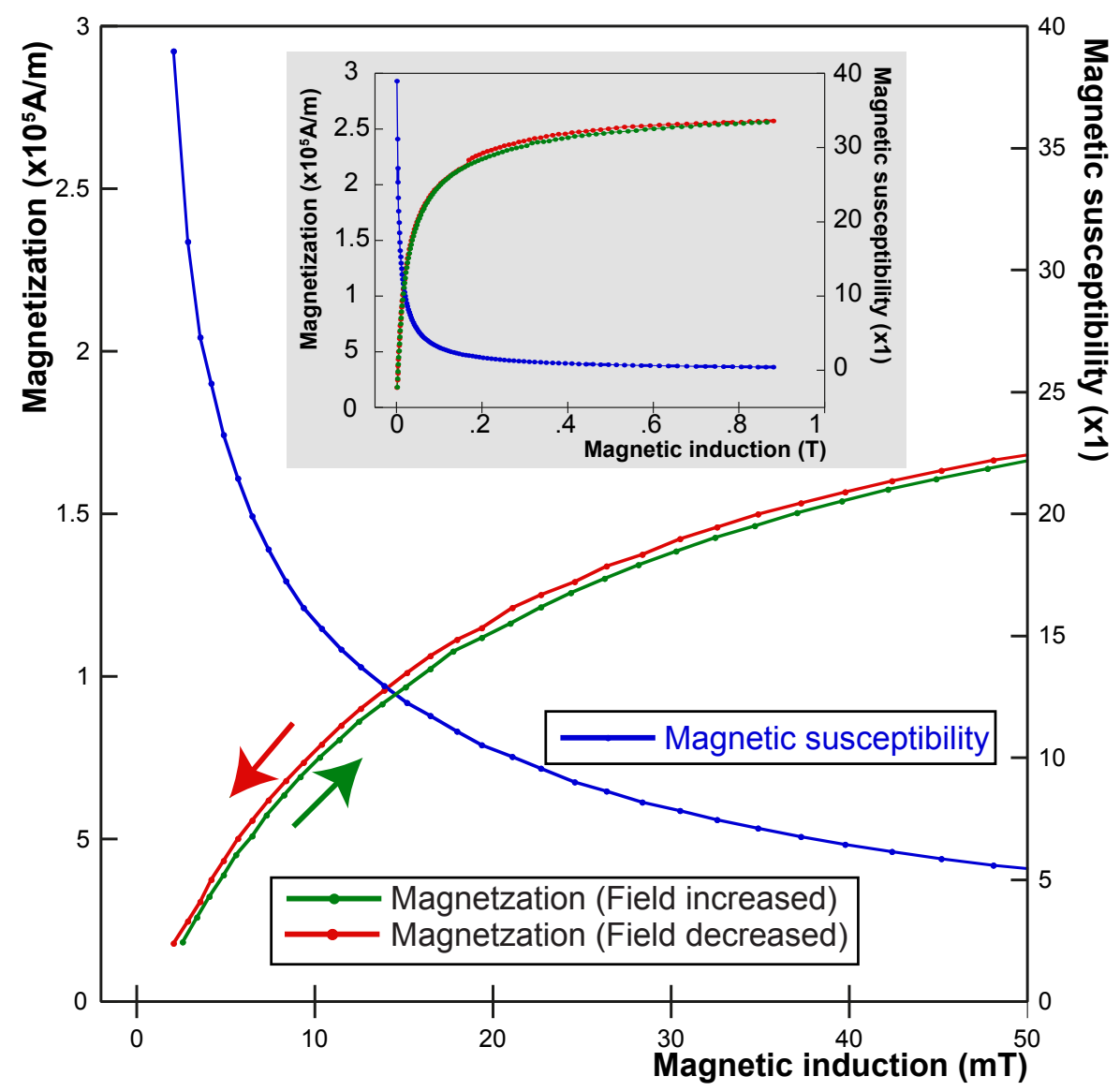

Fig. S2 Magnetization curves of the nanoparticles used to prepare the rods. The magnetization curves $M(H)$ were obtained by vibrating sample magnetometry on a $3.9 \%$ volume fraction ferrofluid suspension of non-aggregated citrated particles. At this low concentration, the magnetic interaction between the particles is negligible and thus, no demagnetizing field takes place. Data have been extrapolated as if the solution volume fraction were 100\%. The main figure is a plot of the magnetization cycle for the low values of the field at which the flexural experiments were performed. Green: field is increased, Red: field is decreased (refer to the left-axis for the scale). Blue data: magnetic susceptibility $\chi^{v}=\frac{M}{H}$ deduced from the magnetization values (scale on the right axis). The full cycle, shown in the grey inset, confirms the known surperparamagnetism of such a suspension [5]. The slight shift between the increasing and decreasing curves ascribes to a known thermal drift of the Hall probe gaussmeter used to measure the magnetic induction. 


\section{ESI, note 1: Optical and Atomic Force Microscopy measures of the rod radius}

Much attention is necessary for the precise measurement of the radius $r$, on which depends the further determination of other parameters such as the Young modulus $\mathrm{Y}\left(Y=\frac{4}{\pi} r^{-4} C\right.$ for cylindrical rods [13]), the magnetic susceptibility $\chi \propto C \cdot r^{-2}$ (ESI $\dagger$, note 2), and hence the particle volume fraction $\phi \propto \chi$. We therefore searched for a precise direct determination of the radius of individual rods. Unfortunately, the typical diameter size $(200-800 \mathrm{~nm})$ was in the order of the Abbe's resolution limit $\rho=\frac{0.61 \lambda}{N A} \sim 250 \mathrm{~nm}$ ( $N A$ is the numerical aperture of the objective and $\lambda$ the wave length). We thus developed a method to measure the rod radius inspired by the measurement of the length of sub-micron large bacteria by fluorescent microscopy [21] and driven by the assumption that in reflection microscopy, individual nanoparticles of the rod behave as individual point sources, like fluorescent particles in biological samples. We neglected the possible interference between them as ascribed from weak coherence of the light source. We also adopted the simple viewpoint where each diffuser reemits light with the same intensity. With the further hypothesis that at a given crosssection the nanoparticle concentration is homogeneous, the back scattered light intensity is simply dictated by the geometry and should be proportional to $2 r_{1} \sqrt{1-\left(\frac{x}{r}\right)^{2}}$, where $x$ denotes for the abscissa along the orthogonal axis to the rod, $r$ the radius in the transverse direction of the optical axis and $r_{1}$ the radius along the optical axis $\left(r_{1} \neq r\right.$ for an elliptic cross-section). In this view, the reflected light is therefore the point spread function of the microscope convoluted by the emitted light and should be: $I_{r}(x)=I_{0}(r) \int_{-r}^{r} J_{1}\left[a(s-x)^{2}\right] 2 r_{1} \sqrt{1-\left(\frac{s}{r}\right)^{2}} d s$ where $J_{1}$ is the Bessel function of the first kind and $a=\frac{2 \pi N A}{\lambda}$. A common simplification consists in replacing $J_{1}$ by a Gaussian law with standard deviation $\sigma_{x y}=.21 \frac{\lambda}{N A}[19]$. To test this model, we thus compared the expected intensity $I_{r}(x)=I_{0}+I_{1} \int_{-r}^{r} \exp \left[-\frac{\left.\left(s-x-x_{0}\right)\right)^{2}}{2 \sigma_{x y}{ }^{2}}\right] 2 r \sqrt{1-\left(\frac{s}{r}\right)^{2}} d s$ with many measures of grey intensity profiles from reflection images of the various rods (Fig. $1 \mathrm{C}$ and ESI $\dagger, \mathrm{S} 2$ ). $I_{0}, I_{1}, x_{0}$ and $r$ where adjustable parameters. In all cases, the theoretical curve fitted well the measured curves. We could thus automatize the procedure to probe the rod along its entire length (every two pixels=91 nm, ESI $\dagger$, Fig. S3).

To check the accuracy of these optical measures, we compared them with measures obtained by an AFM scan performed on the same optically analyzed rod. The AFM used was a MFP-3D-BIO from Azylum Research, mounted on an inverted Olympus optical microscope. The AFM cantilevers were OMCL AC160TS R3 probes from Olympus with nominal stiffness of $26 \mathrm{~N} / \mathrm{m}$, nominal tip height 
of $14 \mu \mathrm{m}$, and nominal tip radius of $7 \mathrm{~nm}$. The resonance frequency was $271 \mathrm{kHz}$ and the scan rate was $1 \mathrm{~Hz}$. The imaging was performed in the soft tapping regime. The images were acquired with lateral sizes ranging from 5 to $20 \mu \mathrm{m}$. To measure the radius by analysis of the optical images, we used the same equipment as for the rest of the experiments (camera, objective and beam splitter). To avoid the difficulties of AFM on water immersed samples, we partially allowed the sample to dry before the scan. It is clear that the procedure somewhat damaged the rods (as shown by images taken before, during and after rehydratation) but we found that a greater variability of the rod cross-section was actually more suitable than homogeneous rods to compare both radius measurement methods. After AFM analysis, the samples were gently rehydrated with a pipette, without moving the sample from the microscope stage. Images taken before and after the procedure showed that no observable modification occurred during the procedure. The AFM scans were done in tapping mode in the soft tapping regime from which we derived the height and radius of the rod profiles from the AFM scans (ESI†, Fig. S3) taking into account the geometry of the tips. The height was found to be smaller than the width diameter, indicating that their lower surface may have flattened on the coverslip while drying. In all cases, we found that the variation of the height and the width were strongly correlated. The model for the optical analysis also holds for elliptic cross-section (or half-cut elliptic section) through the adjustment of the fit variable $I_{1}$.

We performed this analysis respectively on 15 rods made from the $13 \mathrm{~nm}$ (and also $8 \mathrm{~nm}$ ) average diameter nanoparticle fractions. The graphs of ESI $\dagger$, Fig. S3 show the strong similitude between the profiles from the two methods for three rods. However, we find that rather than being stochastic, the sign of the difference between the two curves is persistent over micron-long distances. Our interpretation is that, given the low number of particles (see Table 1 and Fig. 3), the AFM tip scans the polymer hairy shell whereas the optical method accounts for the presence of the inorganic nanoparticles. The AFM precision could thus be affected by the presence of sticky blobs of polymer or nanoparticles onto the tip, or the presence of a heterogeneous water layer around the rod. Despite these differences, we found that the mean difference (averaged over the rod length) between AFM and optical measures were respectively 1.5, 1.9 and $12 \mathrm{~nm}$ and that the standard deviation was ranging from 20 to $30 \mathrm{~nm}$. This latter value of $30 \mathrm{~nm}$ also appeared to be the typical standard deviation of the optical radius measured along a water-immersed rod and was retained to be the uncertainty of our optical measurement. 
ESI, Figure S3: Optical and AFR measurements of the rod radius
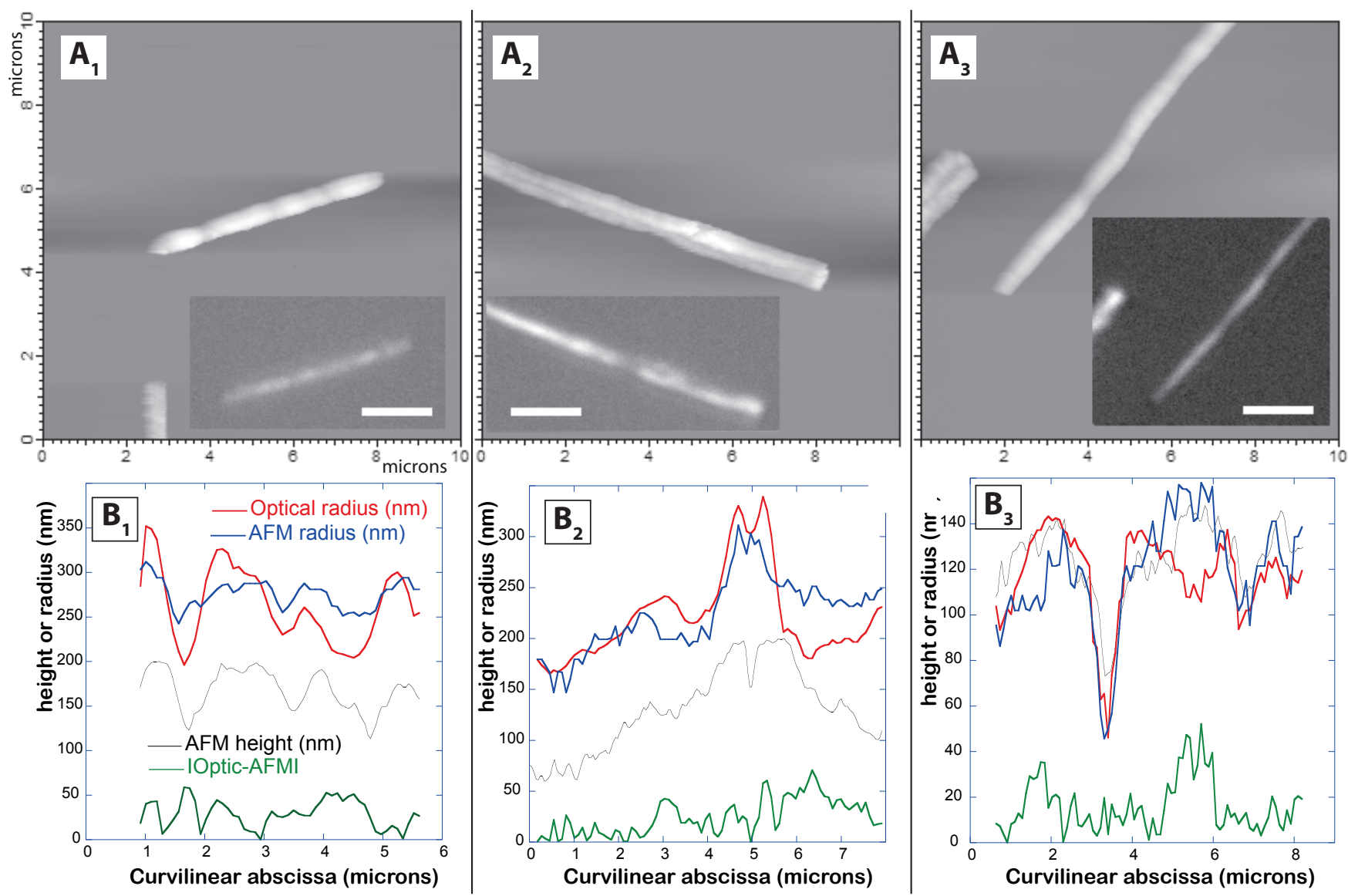

Fig. S3 Comparison of rod radius measurement by AFM and optical reflection image analysis for three different rods. $A_{1}-A_{3}$ grey intensity map of the height measured by AFM. For each panel, the inset shows the reflection optical image of the same rod after the sample has been rehydrated (bars $=2 \mu \mathrm{m}$ ). $\mathrm{B}_{1}-\mathrm{B}_{3}$ each panel corresponds to the $\mathrm{A}$ images and shows the AFM-measured height (black line), the AFM-measured radius deduced from the width (blue line), the radius derived from the optical analysis (red line) and the absolute difference between the two blue and red measures (green line). For each curve the sampling was every 2 camera pixels $=93 \mathrm{~nm}$. 


\section{ESI, note 2: Theory of the magnetoelastic experiments}

In this section, we detail the theoretical model used to deduce the magnetic susceptibly $\chi$ from the magnetoelastic bending of a cantilevered rod submitted to a uniform external field $\vec{B}_{0}=\mu_{0} \vec{H}_{0}$ ( $\mu_{0}$ is the vacuum permeability). As described in the main text, we assume that the rod geometrical parameters - the radius $r$, the length $L$, and the deflection $\delta$ of the rod tip - as well as its bending modulus $C$ are known from independent measurements. As shown on (Fig. 2A), we note $\vec{H}$ the field inside the $\operatorname{rod}$ and $\vec{M}$ its magnetization. The symbols $\|$ and $\perp$ applied to any vector $(\vec{M}$, $\left.\vec{B}_{0}, \vec{H} \ldots\right)$ denote respectively their longitudinal and orthogonal projections on the rod. In absence of remanent field (the rods are superparamagnetic), the bulk rod material is characterized by a magnetic susceptibility which is defined as $\chi(H)=\frac{M}{H}$. We assume the material to be homogeneous and isotropic so that this relationship is the same in any direction and we may write: $\vec{M}=\chi(H) \vec{H} . \S$. In the absence of magnetic dipolar interaction between the nanoparticles [10], we also have $\chi(H)=$ $\frac{\phi}{\phi^{v}} \chi^{v}(H)$ where $\phi$ and $\phi^{v}$ are respectively the volume fractions of the particles in the rod and in the ferrofluid of the same nanoparticles on which the measurement of $\chi^{v}$ was performed by vibrating sample magnetometry (VSM).

We first consider a non-deformable paramagnetic rod of size $L$ and radius $r$, submitted to $\vec{B}_{0}$ oriented at an angle $\alpha=\frac{\pi}{2}-\theta$ with respect to the rod main axis (Fig. 2A). When $L \gg r$, the cylindrical rod may be approximated by a degenerate ellipsoid for which the Maxwell equations have an analytical solution. In our case where the material is uniform and isotropic, the computation yields the demagnetizing fields and the magnetization in both directions: $\vec{M}_{\|(\perp)}=\chi_{\|(\perp)} \vec{H}_{0 \|(\perp)}$ where $\chi_{\|(\perp)}=\frac{\chi\left(H_{\|(\perp)}\right)}{1+n_{\|(\perp)} \chi\left(H_{\|(\perp))}\right.}$ is the effective susceptibility for each canonic directions and $n_{\|(\perp)}$ the respective demagnetization factors. For $L / r \sim 100$, as in the experiments, the infinite cylinder is an excellent approximation which gives $n_{\|}=0$ (there is no demagnetizing field in the axial direction and thus $\left.H_{\|}=H_{0 \|}\right)$ and $n_{\perp}=1 / 2[12]$, with a relative error of $\sim 7 \times 10^{-3}$.

In these conditions, each section of the cylinder is submitted to a magnetic torque per unit

\footnotetext{
${ }^{\S}$ see Landau and Lifchitz, Electrodynamics of continuous media, (Second edition, Pergamon press, Oxford 1984), chapter IV p106 Eq. (29.10) and (29.11)

\see Demagnetizing factors of the General ellipsoid, (1945) Osborn, Phys. Rev. 11 and 12, p351. The calculation holds when the ellipsoid is homogeneous, isotropic (our case) and magnetically linear. Our weaker condition of a non-constant $\chi(H)$ is nevertheless sufficient: in the ellipsoid, a solution exists for any susceptibility $\chi^{\prime}$ in which $\vec{H}$ and $\vec{M}$ are constant throughout the ellipsoid. The solution verifies $M=\chi^{\prime} H$ everywhere. Thus, there is a valid solution for our specific $\chi(H)=\chi^{\prime}=M / H$. By unicity of the solution of the Maxwell equations, it is the only one.
} 
volume $\vec{\Gamma}_{m}=\vec{M} \wedge \overrightarrow{B_{0}}$. The contributions from the $\|$ and $\perp$ yield its algebraic amplitude:

$$
\Gamma_{m}=\Gamma_{m \|}+\Gamma_{m \perp}=\Delta \chi \sin (2 \alpha) \frac{\pi r^{2} B_{0}^{2}}{2 \mu_{0}}
$$

where

$$
\Delta \chi=\chi\left(H_{\|}\right)-\frac{\chi\left(H_{\perp}\right)}{1+\chi\left(H_{\perp}\right) / 2}
$$

depends on both components of the internal magnetic field.

Secondly, we now assume that the cylinder is a Hookean deformable solid of Young modulus $Y$ and bending modulus $C=\frac{\pi}{4} r^{4} Y$. According to classical elasticity equations [13], the restoring torque per unit length of a bent cylinder is $\Gamma_{b}=C \frac{d^{2} \theta}{d l^{2}}$. The local torque balance equation (which may be obtained through minimization of the energy functional even when $\chi$ depends on $H$ [12]) is therefore:

$$
C \frac{d^{2} \theta(l)}{d l^{2}}+\pi r^{2} \frac{\Delta \chi B_{0}^{2}}{2 \mu_{0}} \sin [2 \theta(l)]=0
$$

where $l$ designates the curvilinear abscissa along the rod and $\theta$ the angle with respect to the orthogonal direction to the field (see Fig. 2A). However, this equation relies on the supporting hypothesis of equation (1) which holds for a single straight rod, not for a deformed cylinder that changes direction (i.e. bends) relatively to the external field. It remains approximatively valid ${ }^{l}$ if each section of the rod magnetically responds to the external field independently of what happens in the rest of the rod. We thus designate it as the "independent model". We recently introduced an alternative so-called "axial model" [12], in which we hypothesized that for large $\chi$ the magnetization is mostly longitudinal and also approximatively constant along the rod main axis. In the same paper, using the dipolar approximation, we discussed of the validity of each model and showed that the "independent model" is actually true when $\chi \leqslant 2$ because with this low susceptibility, the main contribution to the internal field $\vec{H}$ in any direction is the external field rather than the magnetic self-induction of the rod. Thanks to the demagnetizing field, this is also true in the transverse direction whatever the value of $\chi>0$. But in the axial direction, quite the contrary happens when $\chi \geqslant 2$ : each cross-section of the rod is influenced by both the close and distant magnetized parts of the rod. As a conclusion, our experiments demonstrated that the "axial model" describes more accurately the shape of nickel wires with $\chi>100$ and also of microrods described here, although $\chi \sim 2$ [12]. It also better predicted the

\footnotetext{
"The bent rod can be approximated by several contiguous ellipsoids.
} 
threshold of the field intensity at which the rod buckles. The constant axial part of the magnetization is determined by the part of the rod which is the most influenced by the external field,i.e. the section most aligned with the external field. In the cantilevered configuration studied here, this is thus the tip of the rod. Using $H_{\|}=H_{0 \|}$ (the demagnetizing factor is assumed to be null along the rod main axis as in the infinite cylinder), this writes $M_{\|}=\chi\left(H_{\|}\right) H_{\|}=\chi\left(H_{\|}\right) \sin [\theta(L)] H_{0}$. We also neglected $\Gamma_{m \perp}$ in front of $\Gamma_{m \|}$ because mathematically $\forall \chi, \chi_{\perp}<2$ when $n_{\perp}=1 / 2$. With these conditions the "independent model" described by equation (3) is replaced by:

$$
C \frac{d^{2} \theta(l)}{d l^{2}}+\pi r^{2} M_{\|} B_{0} \cos [\theta(l)]=0
$$

This "axial model" also presents a very convenient advantage: with the constancy of $M=M_{\|}$, follows the constancy of $\mathrm{H}$ and $\chi$ (see below). But for an accurate measure of $\chi$ we improve for this paper the "axial model" to take into account $\Gamma_{\perp}$ which may account for $\sim 40 \%$ of $\Gamma_{m}$ when $\chi$ is low. If neglected, the experiments described in the main text done for variable field strength and directions (Fig. 2) does not yield constant $\phi$. Since $\vec{H}_{\perp}$ always follows the "independence rule", the expression of $\Gamma_{m \perp}$ in equation (1) is valid and the torque balance of the complemented axial model becomes:

$$
C \frac{d^{2} \theta(l)}{d l^{2}}+\left\{\chi\left(H_{\|}\right) \sin \left(\theta_{L}\right)-\frac{\chi\left(H_{\perp}\right)}{1+\chi\left(H_{\perp}\right) / 2} \sin [\theta(l)]\right\} \cos [\theta(l)] \frac{\pi r^{2} B_{0}^{2}}{\mu_{0}}=0
$$

Without the assumption that $\chi(H)$ is constant, neither equation (3), nor (5) may be integrated. To circumvent these difficulties we performed very small deformation measurements (deflection $\delta \sim$ $L / 50 \sim 1 \mu \mathrm{m})$ in order to keep almost constant the orientation of the rod with respect to the field. In this condition, we approximate in equation (5): $\sin [\theta(l)] \simeq \sin [\theta(L)]^{* *}$. This also allowed to consider $H_{\perp}$ as constant in the rod, and thus also $\chi\left(H_{\perp}\right)$ (Fig. S4). With these approximations, equation (5) may be integrated and yields the shape of the rod:

$$
\left.x(l)=2 \lambda\left\{\sqrt{1-\frac{\sin \left(\theta_{0}\right)}{\sin \left(\theta_{L}\right)}}\right)-\sqrt{1-\frac{\sin [\theta(l)]}{\sin \left(\theta_{L}\right)}}\right\}
$$

\footnotetext{
${ }^{* *} \sin [\theta(l)] \simeq \sin [\theta(L)]+\cos \left(\theta_{L}\right)\left(\theta(l)-\theta_{L}\right)$. With $\theta_{L}-\theta_{0} \lesssim 3^{\circ} \ll \theta_{0}=55^{\circ}$ (see further), the approximation is better than $4 \%$. Alternatively, replacing $\sin [\theta(L)]$ by $\sin [\theta(l)]$ yields the equation of the independent model (Eq. $(3))$. Indeed, with a small deformation, the infinite straight cylinder model holds to compute the internal magnetization. Thus, analytical integration of equation (3) is feasible and yields somewhat different expressions which are: $x(l)=$ $\kappa\left[\arcsin \left(\frac{\sin \theta(l)}{\sin \theta_{L}}\right)-\arcsin \left(\frac{\sin \theta_{0}}{\sin \theta_{L}}\right)\right], y(l)=\kappa\left[\operatorname{arccosh}\left(\frac{\cos \theta_{0}}{\cos \theta_{L}}\right)-\operatorname{arccosh}\left(\frac{\cos \theta(l)}{\cos \theta_{L}}\right)\right]$ and $L=\kappa \int_{\theta_{0}}^{\theta_{L}} \frac{d \theta^{\prime}}{\sqrt{\sin ^{2} \theta_{L}-\sin ^{2} \theta^{\prime}}}$ with $\kappa=\sqrt{\frac{\mu_{0} C}{\pi r^{2} \Delta \chi B_{0}^{2}}}$. Although this model does not match the rod shape as well as the "axial model" [12], they are very similar for small deflections. In this case, both models yield similar values for the volume fraction $\phi$.
} 


$$
y(l)=\lambda \int_{\theta_{0}}^{\theta(l)} \frac{\sin (\theta) d \theta}{\sqrt{1-\frac{\sin (\theta)}{\sin \left(\theta_{L}\right)}}}
$$

with $\lambda=\sqrt{\frac{\mu_{0} C}{2 \pi r^{2} \Delta \chi B_{0}^{2}}}$. From equations (6) and (7), we deduce the relation between $L$ and $\theta_{L}$ :

$$
L=\lambda \int_{\theta_{0}}^{\theta_{L}} \frac{d \theta}{\sqrt{1-\frac{\sin (\theta)}{\sin \left(\theta_{L}\right)}}}
$$

as well as the deflection of the tip:

$$
\delta=-\sin \left(\theta_{0}\right) x(L)+\cos \left(\theta_{0}\right) y(L)
$$

From the experimental measurements of $L$ and $\delta$, we numerically solved equations (8) and (9) to find $\theta_{L}$ and $\lambda$ from which follows $\Delta \chi$. Equation (8) always admits a solution for $\theta_{L} \geqslant \theta_{0}>0$. But in the case $\theta_{0}=0$, a solution exists only if $L \geqslant 2 \lambda$. This is the mathematical translation of the magnetoelastic buckling instability which occurs at the critical field $B_{c}=\frac{1}{r L} \sqrt{\frac{2 \mu_{0} C}{\pi \Delta \chi}} \simeq \frac{1}{r L} \sqrt{\frac{2 \mu_{0} C}{\pi \chi\left(H_{0 \|_{L}}\right)}}$ in the axial model [12].

From $\Delta \chi$ and the geometrical parameters, one can compute $\chi\left(H_{\|}\right)$and $\chi\left(H_{\perp}\right)$, each of them being proportional to the magnetic susceptibility of the nanoparticles which compose the rods, i.e. $\chi\left(H_{\|(\perp)}\right)=\frac{\phi}{\phi^{v}} \chi^{v}\left(H_{\|(\perp)}\right)$. Using the expression of $\Delta \chi$ and the data $\chi^{v}(H), \phi$ could thus be obtained by solving numerically the set of equations:

$$
\begin{gathered}
H_{\|}=H_{0 \|} \quad H_{\perp}=\frac{H_{0 \perp}}{1+\frac{\phi}{\phi^{v}} \chi^{v}\left(H_{\perp}\right) / 2} \\
\phi=\phi^{v} \frac{\Delta \chi}{\chi^{v}\left(H_{0 \|}\right)-\chi^{v}\left(H_{\perp}\right) \frac{H_{\perp}}{H_{0 \perp}}}
\end{gathered}
$$

based on these latter equations, Fig. S4 shows the variation of the magnetic susceptibilities in a infinitely-long cylinder as a function of the field components, which depends themselves on the induction field angle with respect to the cylinder. 
ESI, Figure S4: Rod magnetic susceptibility as a function of the field incidence angle

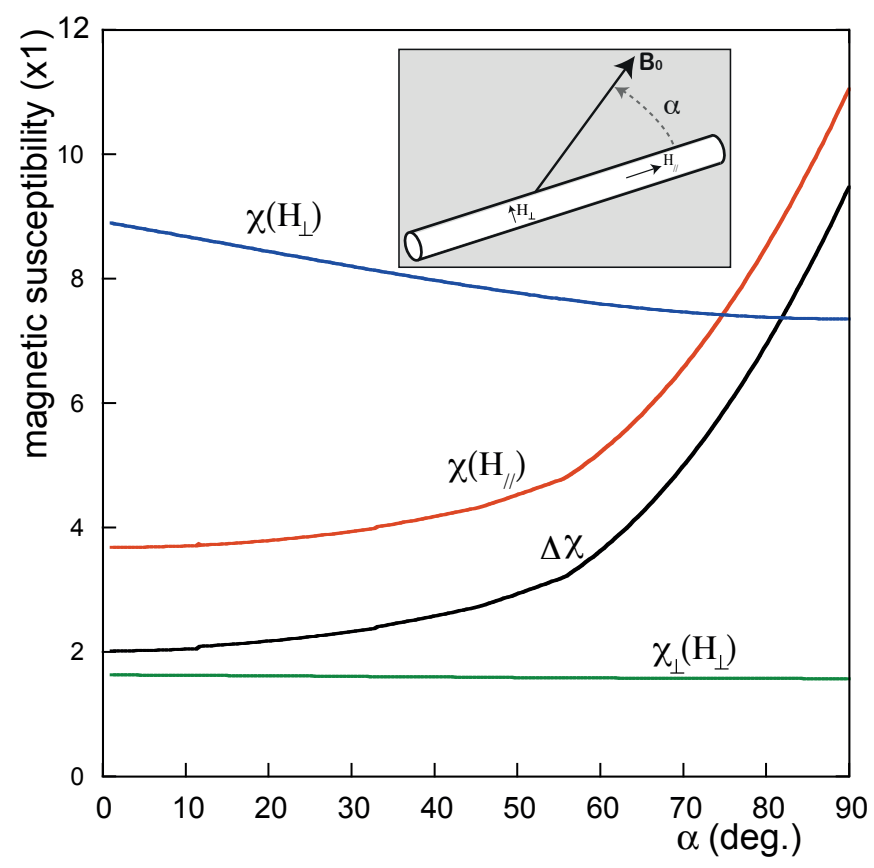

Fig. S4 Rod magnetic susceptibility as a function of the field incidence angle. Computation of the magnetic susceptibilities of an infinitely long rod (with a particle volume fraction of $\phi^{v}=15 \%$ ) as a function of its orientation $\alpha$ in respect with the external induction field $B_{0}=5 \mathrm{mT}$ (see inset). The various susceptibilities are numerically computed from equations (10) and (11) and after the VSM data $\chi^{v}(H) . H_{\|}$and $H_{\perp}$ are respectively the axial and orthogonal components of the magnetic field inside the $\operatorname{rod}, \Delta \chi=\chi\left(H_{\|}\right)-\chi_{\perp}\left(H_{\perp}\right)$ with $\chi_{\perp}=\frac{\chi}{1+\chi / 2}$. Interestingly, $\Delta \chi$ varies dramatically by almost a factor of 5 when $\alpha$ is rotated by $90^{\circ}$ but varies by less than $10 \%$ for $\alpha$ between 0 and $20^{\circ}$. 


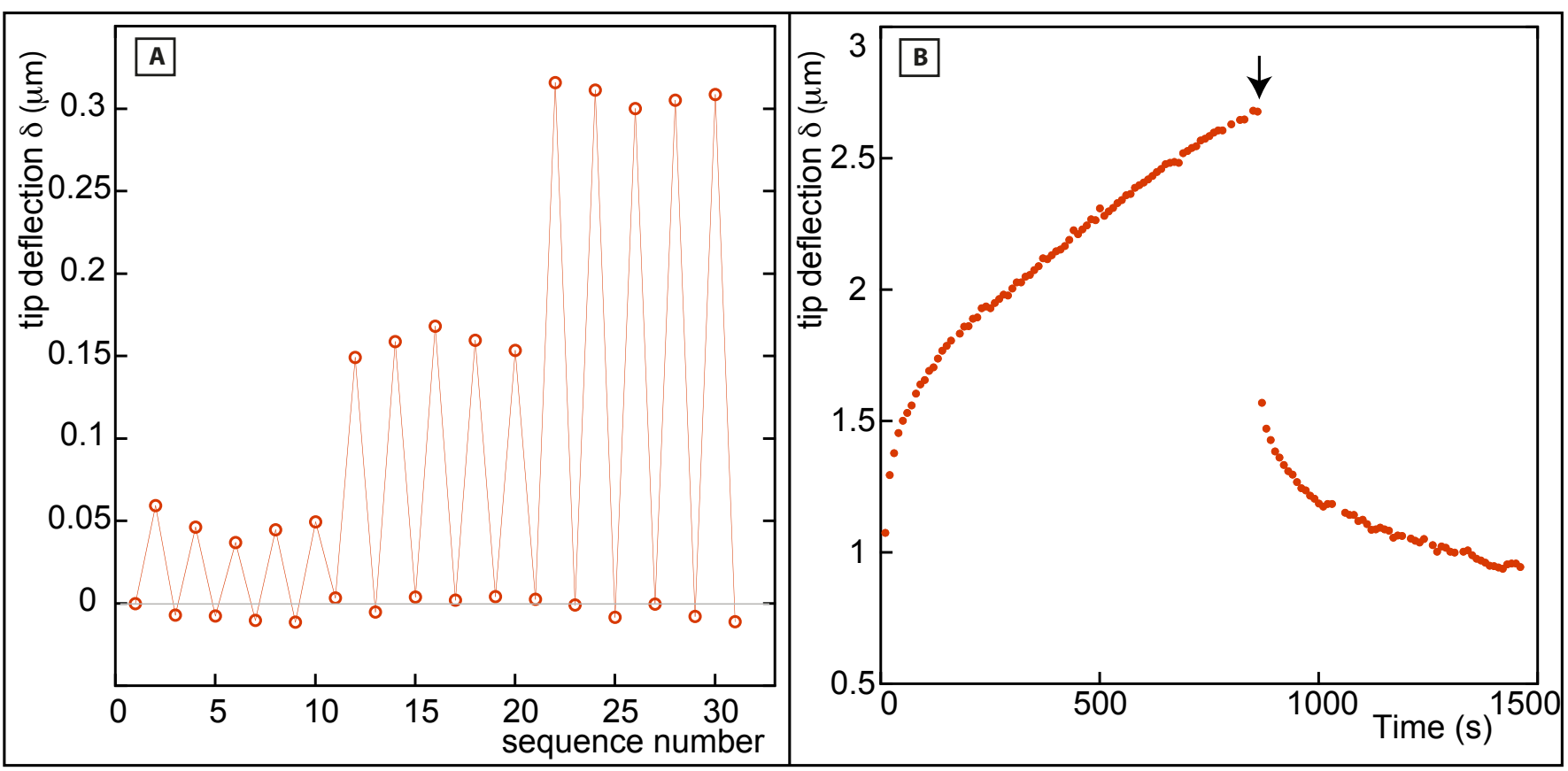

A: deflection distance $\delta$ of a $44.6 \mu \mathrm{m}$ long cantilever rod during a typical sequence of magnetic measurements with an applied induction field. The field is alternatively turned on for $700 \mathrm{~ms}$ (including $200 \mathrm{~ms}$ of camera acquisition) and off (for the same time) to check its elastic return to its undeformed state. The field is always applied at $35^{\circ}$, at increasing strength $(3.26,5.59$ and $7.61 \mathrm{mT})$ and 5 times for each strength to test the reproducibility and average out the variations of the deflected amplitude due to thermal fluctuations. B: by contrast, a strong induction field (33.7 mT) applied for a long time yields large non-elastic deformations. The arrow indicates the time at which the field is turned off. The recording has been stopped but the rod does not return to the initial state $(\delta=0)$ even after hours.

ESI, Movie 1: automatic recognition of the deflected rod centerline by the image recognition software

Movie made of 31 time-laps reflection images of a $53 \mu \mathrm{m}$ long rod deflected by an increasing magnetic field successively turned on and off according to the experimental procedure shown on ESI $\dagger$, Fig. S5. Lower left bar $=10 \mu \mathrm{m}$. The direction of the field is indicated by the upper right arrow $\left(\theta_{0}=35^{\circ}\right)$ and its intensity written on the movie. The dark line drawn on the middle of the rod image is its centerline automatically digitized by our software. 